\title{
Evaluación de la calidad de vegetación ribereña en dos cuencas costeras del sur de Chile mediante la aplicación del índice QBR, como base para su planificación y gestión territorial
}

\section{Riparian vegetation quality evaluation of two coastal watersheds in southern Chile by applying QBR index as base for its territorial management and planning}

\author{
Sebastián Carrasco ${ }^{1 *}$, Enrique Hauenstein ${ }^{1}$, Fernando Peña-Cortés ${ }^{2}$, Carlos Bertrán ${ }^{3}$, Jaime \\ TAPIA ${ }^{4}$ \& LUIS VARGAS-CHACOFF ${ }^{3}$
}

${ }^{1}$ Escuela de Ciencias Ambientales, Facultad de Recursos Naturales, Universidad Católica de Temuco, Casilla 15-D, Temuco, Chile.

${ }^{2}$ Laboratorio de Planificación Territorial, Núcleo de Investigación en Estudios Ambientales, Universidad Católica de Temuco, Casilla 15-D, Temuco, Chile.

${ }^{3}$ Instituto de Ciencias Marinas y Limnológicas, Universidad Austral de Chile, Casilla 567, Valdivia, Chile.

${ }^{4}$ Instituto de Química y Recursos Naturales, Universidad de Talca, Casilla 747, Talca, Chile.

*sebastian.icarrascom@gmail.com

\begin{abstract}
RESUMEN
La vegetación ribereña comprende la zona ecotonal en la transición entre un cuerpo de agua y el ambiente terrestre contiguo, desempeñando un rol elemental en el funcionamiento de los ecosistemas acuáticos. La cobertura, estructura, y composición de la vegetación influyen de manera fundamental en el control de la entrada de energía al ecosistema acuático. Las múltiples funciones ambientales que cumplen los ecosistemas ribereños, y el flujo de servicios ecosistémicos que directa o indirectamente influyen en el bienestar de la sociedad, hacen que el conocimiento de la calidad ecológica de estas áreas sea considerado como un buen indicador ambiental, útil en la planificación y gestión del territorio, especialmente en ecosistemas costeros. Mediante la aplicación del índice QBR se determinó la calidad de la vegetación ribereña en los ríos Lingue y Chaihuín, ambas cuencas costeras del sur de Chile. Los resultados obtenidos indican que el río Chaihuín presenta una vegetación de mejor calidad en sus riberas, en comparación con el río Lingue, donde los bajos valores de QBR son producto de la sustitución de la vegetación ribereña natural para el establecimiento de terrenos agrícolas y plantaciones forestales. La aplicación de este índice y la experiencia global de su utilización indican que resulta ser un instrumento económico y de rápida aplicación, que entrega resultados confiables y útiles para la toma de decisiones en el diagnóstico territorial, considerando las múltiples funciones y valores que hacen de los ecosistemas ribereños buenos indicadores ambientales, diagnosticando los principales impactos y problemas para proponer medidas de restauración y/o mitigación.
\end{abstract}

Palabras clave: Río Lingue, río Chaihuín, QBR, vegetación ribereña.

\begin{abstract}
Riparian vegetation comprises the ecotonal zone in the transition between a water body and the adjacent terrestrial environment and it plays an elemental role in the functioning of aquatic ecosystems. Coverage, structure, and composition of vegetation have a fundamental influence in controlling the energetic input in the aquatic ecosystem. The multiple environmental functions performed by riparian ecosystems and the flow of ecosystem services, influencing in a direct or indirect way the well-being of society, makes knowledge of ecological quality of these areas to be considered as a good environmental indicator. It is useful in territory management and planning, particularly in coastal ecosystems. By applying the QBR index, it was possible to determine the quality of riparian vegetation in Chaihuín and Lingue rivers, both coastal basins in southern Chile. The results indicate that the Chaihuín river has a better quality of vegetation on its banks, compared with Lingue river, where low QBR values are product of the replacement of natural riparian vegetation due to establishment of agricultural land and forest plantations. The application of this index and the overall experience of its use, indicate it to be an economic and quick-use tool that provides reliable and useful results for decision-making in territorial diagnosis, considering the multiple roles and values that makes riparian vegetation good environmental indicators and diagnosing main impacts and problems to propose restoration and/or mitigation measures.
\end{abstract}

KEYwords: Lingue river, Chaihuín river, QBR, riparian vegetation. 


\section{INTRODUCCION}

Las comunidades vegetales ribereñas son las que se desarrollan adyacentes a los ecosistemas acuáticos y mantienen la integridad ecológica de éstos (Rzedowski 1994, Norris 2001, Ceccon 2003). Al ser ecotonos entre ambientes acuáticos y terrestres (Mitsch et al. 1994, Ceccon 2003), poseen características singulares y desempeñan funciones primordiales para el óptimo funcionamiento de los humedales (Fernández et al. 2009). De las múltiples funciones que cumplen estos ecosistemas, se derivan una serie de servicios ecosistémicos beneficiosos para el bienestar social, entre ellos los servicios de PRODUCCIÓN (de materias primas, recursos genéticos, recursos medicinales, recursos ornamentales), de REGULACIÓN (hídrica, microclimática, retención y formación edáfica, buffer biológico), de INFORMACIÓN (estética, recreativa, turística, cultural, espiritual, histórica, científica y educacional) y de HÁBITAT (alimento, refugio, agua y territorio para la reproducción), entre otros (Carothers 1977, Nahuelhual \& Núñez 2011). En materia de conservación, la vegetación ribereña destaca como un ecosistema de alta productividad primaria y que constituye hábitat de fauna tanto acuática como terrestre con problemas de conservación (e.g. Lontra provocax Thomas 1908, Rhinoderma darwinii Duméril \& Bibron 1841). Actualmente, las áreas ribereñas están dentro de los ecosistemas más amenazados a nivel global, lo que se evidencia a través de la degradación o pérdida de la cobertura vegetal, generando la degradación de los cuerpos de agua y afectando sustancialmente las funciones, servicios y bienes que estos ecosistemas proporcionan (Bunn et al. 1999, Muotka \& Laasonen 2002). De lo anterior se desprende que las áreas ribereñas constituyen sitios prioritarios para la conservación de la naturaleza debido a su alto grado de amenaza contrastado con su importancia para la conservación de la diversidad biológica, funciones ambientales y provisión de servicios ecosistémicos. Las múltiples funciones ambientales de la vegetación ribereña realzan su utilidad como un buen indicador en la gestión y planificación territorial, permitiendo su inclusión como elemento clave para la calificación del estado ecológico de los ecosistemas acuáticos (Suárez et al. 2002). Las razones son evidentes y obedecen, por una parte, a sus valores naturales (elevada diversidad biológica) (Girel \& Manneville 1998, Ward 1998), a la heterogeneidad del paisaje y a su capacidad para incidir sobre la calidad ambiental del ecosistema acuático que rodea, a través del control de la temperatura del agua (Beschta et al. 1987), de la entrada de materia orgánica y de los nutrientes que aportan las fuentes difusas (Fischer \& Likens 1973, Schade et al. 2001, 2002), e incluso por su capacidad para diseñar microambientes terrestres (Brosofske et al. 1997). Con el objetivo de determinar la calidad de la vegetación ribereña en dos sistemas lóticos costeros del sur de Chile, mediante un análisis eficaz y de bajo costo que sea útil en la planificación territorial, se aplicó el índice QBR (qualitat del bosc de ribera=calidad del bosque de ribera) (Munné et al. 1998a, 1998b, 2003) el que ha sido empleado y ha entregado buenos resultados en ríos españoles (Ibero et al. 1996, Munné et al. 1998a, 1998b, Prat et al. 1999, Carrascosa \& Munné 2000, Suárez \& Vidal-Abarca 2000, Bonada et al. 2002, Suárez et al. 2002, Munné et al. 2003, Martínez \& Lozano 2004,), portugueses (Moreira 2006), turcos (Tüzün \& Albaryak 2005), norteamericanos (Colwell 2007), ecuatorianos y peruanos (Acosta et al. 2009), argentinos (González 2007, Kutscher et al. 2009), mexicanos (Rodríguez-Téllez et al. 2012) y chilenos (Palma et al. 2009, Fernández et al. 2009, Peredo et al. 2012). En este contexto, el presente estudio evalúa la calidad de la vegetación ribereña en dos ríos del borde costero del sur de Chile, además, se evalúa la efectividad del índice QBR como indicador ambiental y su utilidad en la planificación y gestión territorial.

\section{MATERIALES Y METODOS}

Área de ESTUdio

El área de estudio consideró las riberas de los ríos Lingue y Chaihuín, ambos pertenecientes administrativamente a la región de Los Ríos, sur de Chile (Fig. 1). El río Lingue posee una longitud aproximada de $58 \mathrm{~km}$, desemboca al sur de la bahía de Maiquilahue, en la localidad de Mehuín (39²6’S, $73^{\circ} 13^{\prime} \mathrm{W}$ ) y su cuenca posee una superficie de $319,93 \mathrm{~km}^{2}$. El río Chaihuín por su parte, tiene una longitud de 87,7 $\mathrm{km}$, y su cuenca una superficie de $313 \mathrm{~km}^{2}$ desembocando en la localidad homónima (3956'S, 73³4'W). El clima corresponde al tipo templado lluvioso, el cual se caracteriza por altas precipitaciones (entre 2.000 y $2.500 \mathrm{~mm}$ anuales) y baja oscilación térmica producto de la influencia marina (temperatura media anual cercana a $\operatorname{los} 12^{\circ} \mathrm{C}$, llegando a los $18^{\circ} \mathrm{C}$ en los meses más cálidos). Ambos ríos nacen en la Cordillera de la Costa ( \pm 150 m s.n.m.), y sus cuencas son de régimen pluvial, las que por su morfología y pendientes en su porción inferior reciben aportes de caudal provenientes del mar, lo que origina ambientes de tipo estuarino. La vegetación dominante está representada por comunidades de bosque pantanoso, Bosque Laurifolio de Valdivia y Bosque Caducifolio del Sur (Gajardo 1995, Luebert \& Pliscoff 2006).

\section{ÍNDICE QBR}

Se aplicó, sin modificaciones, el índice QBR (Munné et al. 1998a, 1998b, 2003) para evaluar el estado y la calidad de la vegetación ribereña en ambos cursos de agua, este índice pone énfasis en cuatro aspectos fundamentales de los sistemas ribereños: (1) grado de cobertura vegetal, (2) estructura de la vegetación, (3) calidad de la cubierta vegetal y (4) grado de naturalidad del canal fluvial. La cobertura de 
la vegetación evalúa la conectividad entre el área ribereña y los ecosistemas terrestres adyacentes, para ello se consideró el porcentaje de cobertura de árboles, arbustos y helófitas, en conjunto con la conexión que tenga la comunidad terrestre adyacente. La estructura vegetacional evalúa la complejidad estructural del ecosistema ribereño, considerando que la heterogeneidad ambiental puede aumentar su biodiversidad. Para evaluarla se consideró el porcentaje de presencia de cada grupo funcional y la presencia de plantaciones y parches aislados dentro de la zona ribereña. La calidad de la vegetación, permite determinar la naturalidad de las formaciones vegetales presentes, valorada a partir del número de especies nativas en el sector de muestreo, y dependiente del tipo geomorfológico de la zona ribereña. El grado de naturalidad del canal fluvial consideró principalmente las modificaciones antrópicas realizadas al cauce, las que modifican, alteran y perturban el hábitat ribereño. Cada sección fue evaluada de manera independiente en cada estación de muestreo, valorada con una puntuación de 0 a 25 , y la sumatoria de la puntuación de los cuatro apartados fluctuó entre 0 y 100. Los valores de calidad resultantes se distribuyen en cinco rangos: calidad muy buena, estado natural ( $Q B R \geq 90$ ); calidad buena, vegetación ligeramente perturbada (QBR 75-90); calidad intermedia e inicio de alteraciones importantes (QBR 55-70); mala calidad, alteraciones importantes (QBR 30-50); y calidad pésima, degradación extrema $(\mathrm{QBR} \leq 25)$. Los protocolos y formatos para la aplicación del índice se encuentran en JáimezCuéllar et al. (2002). Para calcular el QBR, en cada estación de muestreo se escogió un tramo de $100 \mathrm{~m}$ de longitud (con una separación promedio de $1 \mathrm{~km}$ ), y se consideró toda la anchura potencial del bosque y/o vegetación ribereña. Se delimitó visualmente la orilla (zona del cauce inundable en crecidas periódicas en un periodo aproximado de dos años) y la ribera (zona inundable en crecidas de gran magnitud, periodos de hasta 100 años). En el caso del río Lingue, se aplicó el QBR en 22 estaciones de muestreo, y en el río Chaihuín se obtuvieron datos en 14 estaciones. Cada punto de muestreo se identificó con un número romano, partiendo desde la parte alta de la cuenca, avanzando aguas abajo.

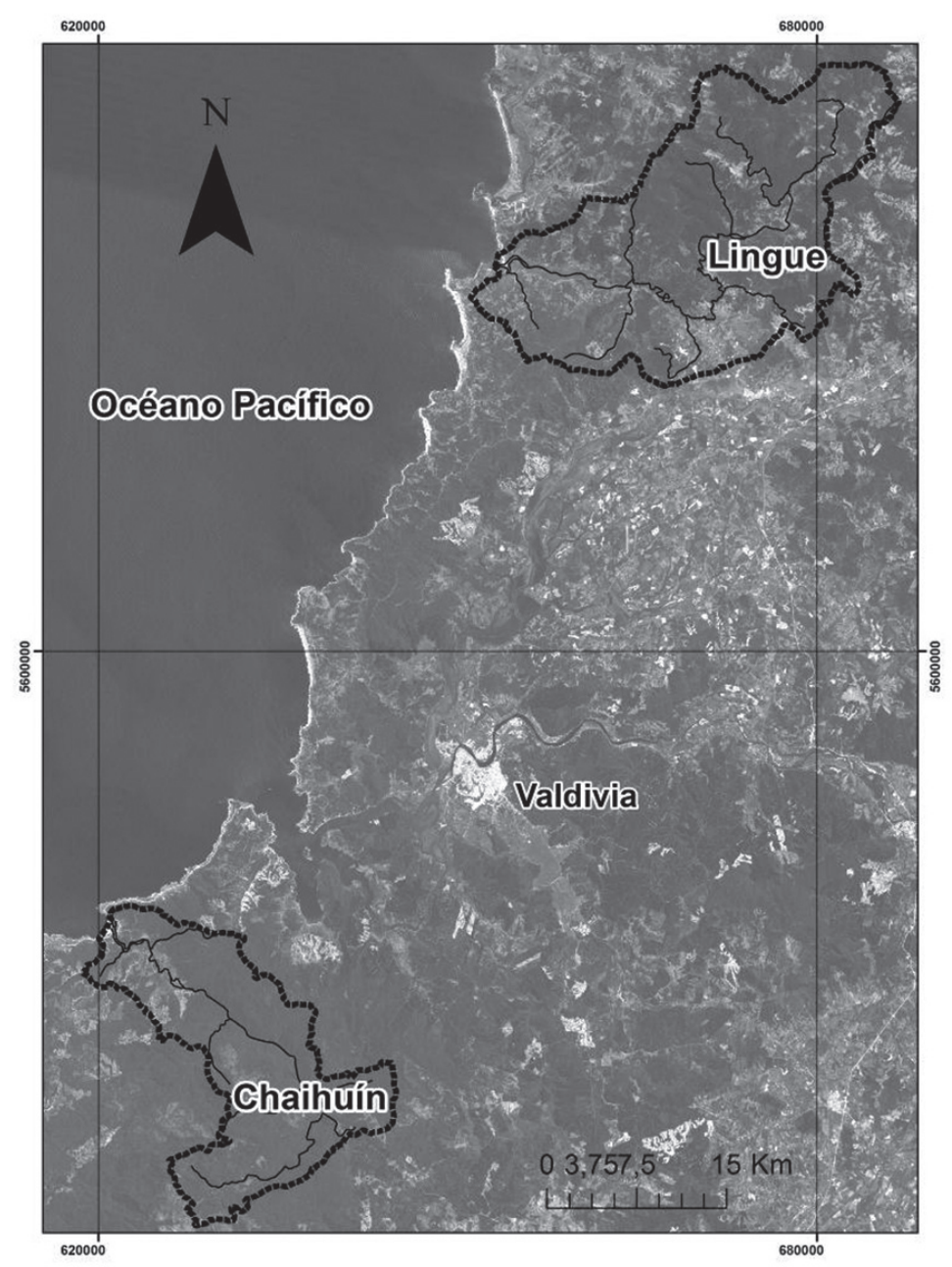

Figura 1. Área de estudio. Cuencas hidrográficas de los ríos Lingue y Chaihuín, región de Los Ríos, Sur de Chile.

FigURE 1. Study area. Watersheds of the Lingue and Chaihuín rivers, Los Ríos region, South of Chile. 


\section{RESULTADOS}

En ambas áreas de estudio se registraron los cinco rangos de calidad en los que se divide el índice QBR, en 22 estaciones de muestreo en el caso del río Lingue, y en 14 estaciones en el río Chaihuín (Fig. 2). En el río Lingue, las estaciones X y XI localizadas en el sector medio de la cuenca presentaron una calidad muy buena, y vegetación ribereña en condiciones inalteradas. En las estaciones I, VI, IX, XVII, XVIII, XX y XXI la vegetación ribereña del río Lingue presentó la peor calidad según el QBR, ubicándose éstas en las secciones alta y baja de la cuenca hidrográfica (Fig. 3). En el caso del río Chaihuín (Fig. 4), las estaciones II, III y IV ubicadas en la zona alta del río presentaron una vegetación ribereña en estado natural, y sólo en una estación (XIV) se encontró una vegetación ribereña de calidad pésima, en el punto de muestreo más cercano a la desembocadura del río. Los resultados obtenidos indican diferencias entre ambas cuencas bajo estudio, relacionándose en el río Lingue, los peores rangos de calidad con las zonas altas y bajas de la cuenca, encontrándose en su sección media la mejor calidad. En el Chaihuín en cambio, los niveles de calidad de vegetación ribereña van disminuyendo a medida que se avanza aguas abajo, hasta la zona estuarina.

En ambas cuencas existe una relación entre los bajos valores de QBR y la actividad humana en las riberas, ya que en los puntos peor evaluados, la actividad antrópica se evidencia en bajos porcentajes de cobertura vegetal, presencia de especies introducidas (e.g. Ulex europaeus L., Rubus constrictus P.J. Müll. \& Lefèvre), establecimiento de plantaciones forestales de pino radiata (Pinus radiata D. Don), eucalipto (Eucalyptus globulus Labill.), y álamo (Populus deltoides W. Bartram ex Marshall), e intervenciones que modifican la estructura y conectividad de las riberas y los sistemas acuáticos (extracción de áridos, puentes, caminos y canalizaciones).

Las especies de flora nativa más representativas de la vegetación ribereña en ambas cuencas fueron, en el estrato arbóreo Nothofagus dombeyi (Mirb.) Oerst., Nothofagus obliqua (Mirb.) Oerst., Drimys winteri J.R. Forst. \& G. Forst., Myrceugenia exsucca (DC.) O. Berg, Blepharocalyx cruckshanksii (Hook. \& Arn.) Nied., Luma apiculata (DC.) Burret, Amomyrtus luma (Molina) D. Legrand \& Kausel, Podocarpus salignus D. Don, y Laurelia sempervirens (Ruiz \& Pav.) Tul. En el estrato arbustivo, destacó la presencia de las especies nativas Berberis microphylla G. Forst., Berberis darwinii Hook., Ugni molinae Turcz., Fuchsia magellanica Lam., Chusquea quila Kunth, y Escallonia rubra (Ruiz \& Pav.) Pers. Las especies introducidas más frecuentes fueron Eucalyptus globulus, Populus deltoides, Pseudotsuga menziesii (Mirb.) Franco, Pinus radiata (en forma de monocultivos establecidos en la orilla y ribera), Rubus constrictus, Acacia dealbata Link., Acacia melanoxylon R. Br., y Ulex europaeus.



FIgURA 2. Distribución de los valores de QBR de cada estación de muestreo en los ríos Lingue y Chaihuín. Linea punteada indica los cortes de las categorías del QBR.

FIGURE 2. Distribution of QBR values for each sampling station in Lingue and Chaihuín rivers. Dotted line indicates cuts of QBR categories. 


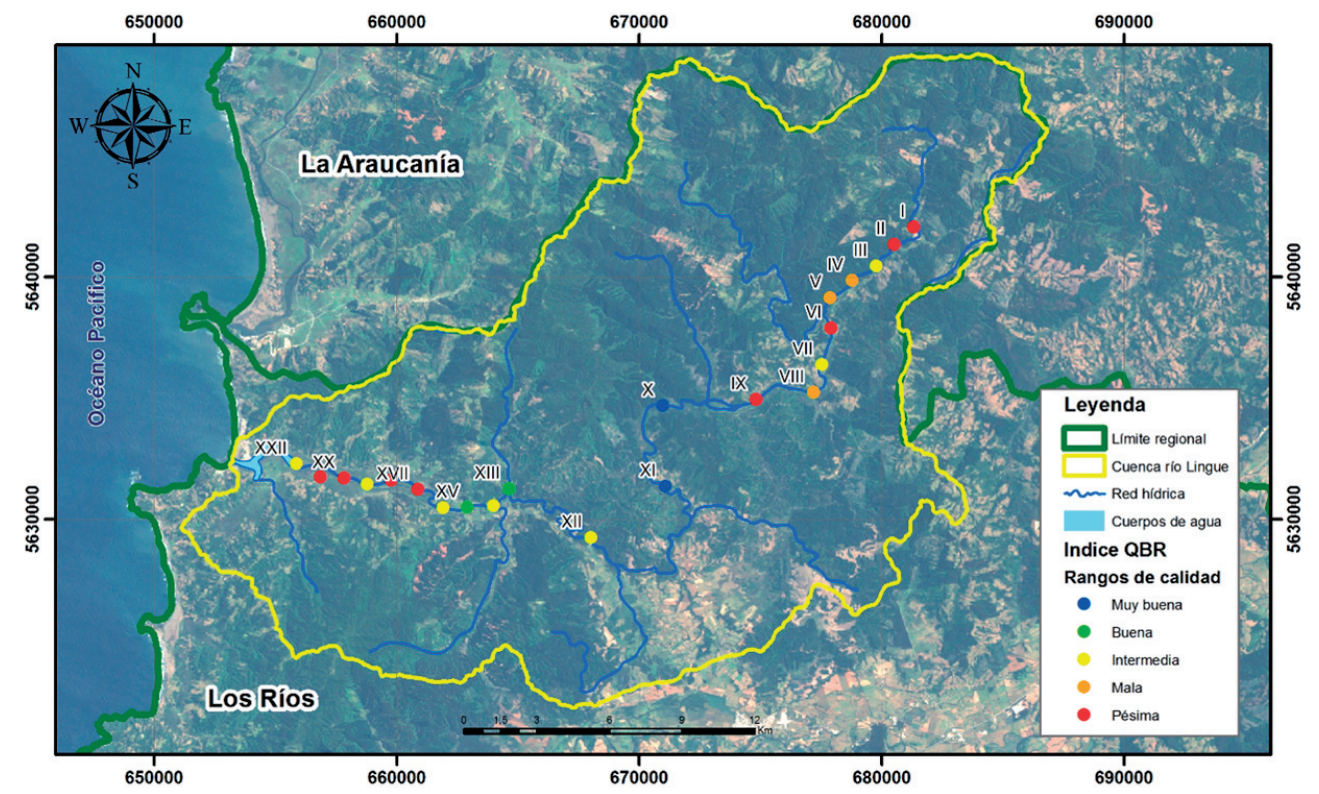

Figura 3. Cuenca del río Lingue y estaciones de muestreo con su rango de calidad de vegetación ribereña.

FIGURE 3. Lingue river watershed and sampling stations with their riparian vegetation's range of quality.

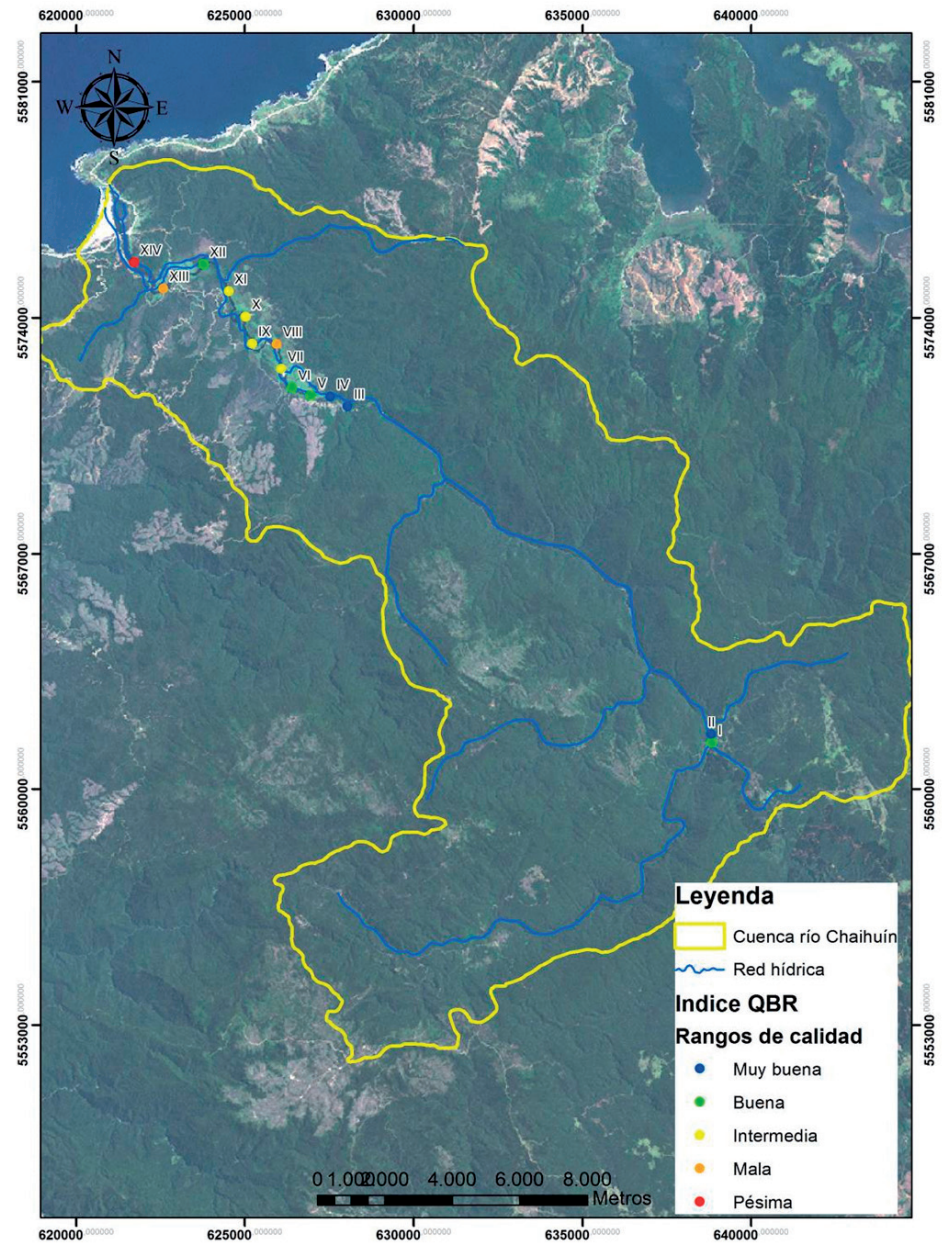

Figura 4. Cuenca del río Chaihuín y estaciones de muestreo con su rango de calidad de vegetación ribereña.

FIGURE 4. Chaihuín river watershed and sampling stations with their riparian vegetation's range of quality. 


\section{DISCUSIÓN}

Los resultados obtenidos de la aplicación del QBR permiten conocer el estado actual de la vegetación ribereña y realizar una aproximación a una comparación de las condiciones ecológicas en los ríos Lingue y Chaihuín; sin embargo, la diferencia en el número de estaciones de muestreo y la distribución de ellas en las cuencas, producto de la limitada accesibilidad en algunos tramos de los ríos, es un factor que dificulta realizar comparaciones de alta confiabilidad en estudios de esta naturaleza. En estos casos, se sugiere complementar el índice QBR con instrumentos de análisis espacial, sistemas de información geográfica (SIG) y sensores remotos que permitan inferir las características ecológicas de las áreas ribereñas.

La distribución espacial de los valores del QBR en el río Lingue, coinciden con los encontrados por Fernández et al. (2009) en el río Maullín (región de Los Lagos). En ambos casos, el QBR arroja mejores rangos de calidad en la zona media de la cuenca, disminuyendo los valores en los extremos.

Ibero et al. (1996) señalan que la degradación de las riberas es más intensa en las partes bajas de los ríos que en las cabeceras, debido a la mayor presión antrópica en estas zonas. Lo anterior concuerda con los resultados obtenidos en el presente estudio para el río Chaihuín, y con lo reportado por Palma et al. (2009) para el estero Nonguén en la región del Biobío.

La baja puntuación del QBR encontrada en algunas estaciones de muestreo de ambos ríos, es resultado de las múltiples intervenciones antrópicas existentes en los ecosistemas ribereños, entre los que destacan las plantaciones forestales, la habilitación de terrenos para la agricultura, infraestructura caminera y abundancia de especies exóticas invasoras. Resultados publicados por Tüzün \& Albaryrak (2005) indican que las zonas calificadas como de peor calidad por el QBR corresponden a las que están más próximas a asentamientos humanos e infraestructura caminera, lo que es concordante con la situación observada en el presente estudio, ya que la proximidad de los ecosistemas ribereños con centros poblados, usos de suelo con fines productivos y la correspondiente infraestructura caminera causan una modificación y fragmentación de los hábitats ribereños, resultando bajas calificaciones de QBR. Dicha situación se constituye en un punto de especial preocupación para la planificación y gestión de estos espacios, considerando que la fragmentación de los hábitats tiene efectos negativos sobre la viabilidad de las semillas y plántulas, afectando directamente la reproducción vegetal y la estructura, composición y funcionamiento de los ecosistemas.

Especialmente relevante en el efecto que se genera en la calidad de estos ecosistemas es la fragmentación de los bosques pantanosos, ya que son ecosistemas únicos, de relevancia mundial y prácticamente desconocidos en Chile desde el punto de vista de su dinámica hidrológica (CorreaAraneda et al. 2011) proporcionan servicios ecosistémicos de importancia para la conservación de la biodiversidad, abastecimiento de agua, diversidad paisajística, belleza escénica, importancia cultural y son muy efectivos en la amortiguación hidráulica de las inundaciones. A pesar de lo anterior, estos ecosistemas están dentro de los más susceptibles a las perturbaciones a escala de cuenca (PeñaCortés et al. 2006).

La importante presencia de plantaciones forestales establecidas en áreas ribereñas, especialmente en el sector alto del río Lingue, sumado a la presencia de caminos forestales, se traduce en bajas puntuaciones de QBR. Esto evidencia algunas de las nefastas consecuencias del establecimiento de monocultivos forestales sin mayor regulación o con deficiente manejo, donde se ven amenazados ecosistemas cuya funcionalidad y calidad ecológica es fundamental a nivel de cuenca para la provisión de servicios ecosistémicos hacia la comunidad. Por otra parte, las especies introducidas invasoras (e.g. Ulex europaeus, Rubus constrictus, Acacia dealbata) constituyen una reconocida amenaza a la biodiversidad, y su presencia altera características ambientales fundamentales, como la identidad taxonómica del ecosistema, sus propiedades estructurales, los ciclos de nutrientes, la productividad primaria y las interacciones bióticas, entre otros (Quiroz et al. 2009, Fuentes-Ramírez et al. 2011).

La pérdida de vegetación natural y la homogeneización estructural de los sistemas ribereños contribuyen a erosionar el área ribereña, ya que los cambios de uso de suelo, el establecimiento de plantaciones forestales, terrenos agrícolas, construcción de viviendas, caminos y puentes dejan el suelo vulnerable a la erosión (Ledger 1968, Andrus 1986, Showers 1996, Pimentel \& Kounang 1998, Harden 2001), además, el QBR penaliza la presencia de infraestructura caminera que modifica sustancialmente el grado de naturalidad del canal fluvial, actúan como rutas de invasión para especies exóticas, funcionando también como barreras físicas que alteran los patrones de distribución de la diversidad biológica.

Considerando la vegetación ribereña y su calidad ecológica como un indicador ambiental de utilidad en la planificación del territorio a escala de cuenca, la mala calidad de la vegetación ribereña que presenta el río Lingue en su cabecera, es una situación compleja que necesita de mayor atención, ya que las zonas altas de los ríos son áreas de importancia vital para el suministro de servicios ecosistémicos, como la mantención de la estabilidad ecológica de los ecosistemas lóticos, el abastecimiento y disponibilidad de agua en la cuenca. Una adecuada planificación territorial, podría considerar las áreas ribereñas como zonas de protección, donde las estrategias de manejo se enfoquen a mantener el equilibrio ecológico de los ríos, humedales y hábitats ribereños, el control de 
riesgos naturales, atenuación de los efectos destructores de las inundaciones (Decamps 1996) y asegurar el óptimo funcionamiento del ecosistema acuático en su totalidad (Tockner \& Ward 1999). Por otra parte, la diversidad florística es importante en los ecosistemas debido a que genera procesos ecológicos funcionales que mantienen el equilibrio y estabilidad de éstos, y es uno de los aspectos poco estudiados, en el que es posible profundizar en el estudio de la vegetación ribereña, sus amenazas y las oportunidades para su conservación.

La aplicación del QBR en el espacio costero de los ríos Lingue y Chaihuín, y la experiencia global en su utilización, indica que éste resulta ser un instrumento económico y de rápida aplicación, que entrega resultados confiables y útiles para la toma de decisiones en el diagnóstico territorial, con el objetivo de conocer el estado de los ecosistemas ribereños, considerando las múltiples funciones y valores que hacen de estos sistemas buenos indicadores ambientales, diagnosticando los principales impactos y problemas ambientales para proponer medidas de restauración y/o mitigación.

Es importante tener en cuenta que el índice puede requerir de ajustes al aplicarlo en otros ecosistemas ribereños, considerando variaciones en altitud, clima y geomorfología de las áreas bajo estudio. En el caso de la experiencia desarrollada por Carrascosa \& Munné (2000) el índice fue utilizado en ríos de alta montaña, concluyendo que el QBR no es aplicable por sobre los $1500 \mathrm{~m}$ de altitud y que por sobre los $800 \mathrm{~m}$ de altitud se requieren algunas modificaciones. De igual forma, Suárez \& Vidal-Abarca (2000) coinciden en la necesidad de realizar ajustes en el caso de ser aplicado en ríos efímeros. En el caso del presente estudio, el índice parece ser idóneo y arrojar resultados confiables sin necesidad de realizar modificaciones.

La aplicación del índice QBR para determinar la calidad de la vegetación ribereña proporciona una aproximación al conocimiento del estado de conservación de los ecosistemas ribereños costeros y su biodiversidad, aspecto fundamental al momento de tomar decisiones de manejo ambiental (Noss 1990).

\section{AGRADECIMIENTOS}

Al proyecto FonDECYT 1110798 "Determinación de indicadores geográfico ambientales y de riesgo natural: Herramientas de soporte decisional para la planificación y gestión territorial en sistemas costeros".

\section{BIBLIOGRAFÍA}

Acosta, R., B. Ríos, M. Rieradevall \& N. Prat. 2009. Propuesta de un protocolo de evaluación de la calidad ecológica de ríos andinos (CERA) y su aplicación a dos cuencas en Ecuador y Perú. Limnetica 28(1): 35-64.

ANDRUS, C. 1986. The economics of erosion control in a subtropical watershed: comment. Land Economics 62: 329-330.

Beschta, R.L., R.E. Bilby, G.W. Brown, L.B. Holtby \& T.D. HoFstra. 1987. Stream temperature and aquatic habitat: fisheries and forestry interactions. In: E.O. SALO \& T.W. Cundy (eds), Streamside Management: Forestry and fishery interactions. Institute of forest resources, University of Washington: Seattle, WA. 191-232.

Bonada, N., N. Prat, A. Munné, M. Plans, C. Solá, M. Álvarez, I. Pardo, G. Moya, G. Ramon, M. Toro, S. Robles, J. Casas, A. Sánchez-Ortega, P. Jáimez-Cuellar \& J. ALBA-TERCEDOR. 2002. Intercalibración de la metodología GUADALMED. Selección de un protocolo de muestreo para la determinación del estado ecológico de los ríos mediterráneos. Limnetica 21: 13-33.

Brosofske, K.D., J. Chen, R.J. Naiman \& J.F. Franklin. 1997. Effects of harvesting on microclimatic gradients from streams to uplands in western Washington, USA. Ecological Applications 7: 1188-1200.

Bunn, S.E., P.M. Davies \& T.D. Mosisch. 1999. Ecosystem measures or fiver health and their response to riparian and catchment degradation. Freshwater Biology 41: 333-345.

CARothers, S.W. 1977. Importance, preservation, and management of riparian habitats: an overview. In: R.R. Jonson \& D.A. JONES (eds). Importance, preservation, and management of riparian habitats: a symposium, pp. 2-4 USDA forest service general technical report RM-43. US Government print office, Washington, DC.

Carrascosa, V. \& A. Munné. 2000. Qualificació dels boscos de ribera andorrans. Adaptació de l'index QBR als d'alta muntanya. Habitats-Centre de biodivesitat (IEA) 1: 4-13.

CecCon, E. 2003. Los bosques ribereños y la restauración y conservación de las cuencas hidrográficas. Ciencias 72 : 46-53.

Colwell, S. 2007. The application of the QBR index to the riparian forest of central Ohio streams. Honor's thesis. School of environment and natural resources, the Ohio State University, Ohio, USA. 44 pp.

Correa-Araneda, F., J. Urrutia, \& R. Figueroa. 2011. Estado del conocimiento y principales amenazas de los humedales boscosos de agua dulce de Chile. Revista Chilena de Historia Natural 84: 325-340.

DeCAMPS, H. 1996. The renewal of floodplain forests along rivers: a landscape perspective. Verhandlungen der Internationalen Vereinigung für Theoretische und Angewandte Limnologie 26: 35-59.

Fernández, L., J. Rau \& A. Arriagada. 2009. Calidad de la vegetación ribereña del río Maullín ( $\left.41^{\circ} 28^{\prime} \mathrm{S} ; 7^{\circ}{ }^{\circ} 59^{\prime} \mathrm{O}\right)$ utilizando el índice QBR. Gayana Botánica 66(2): 269278.

Fisher, S.G. \& G.E. LiKens. 1973. Energy flow in Bear Brook, New Hampshire: an integrative approach to stream ecosystem metabolism. Ecological Monographs 43: 421-439.

Fuentes-Ramírez, A., A. Pauchard, L.A. Cavieres \& R.A. García. 2011. Survival and growth of Acacia dealbata vs. native trees across an invasion front in south-central Chile. Forest Ecology and Management 261: 1003-1009.

Gajardo, R. 1995. La vegetación natural de Chile. Clasificación y 
distribución geográfica. Editorial Universitaria, Santiago, Chile. 165 pp.

Girel, J. \& O. Manneville. 1998. Present species richness of plant communities in alpine stream corridors in relation to historical river management. Biological Conservation 85: 21-33.

GonzÁLEZ, L.M. 2007. Evaluación de la calidad ecológica del hábitat ripario en tramos del río Muerto, Yerba Buena, Tucumán, Argentina. Tesis de Licenciatura en Ciencias Biológicas. Universidad Nacional de Tucumán, Argentina. $98 \mathrm{pp}$.

Harden, C.P. 2001. Soil erosion and sustainable mountain development: experiments, observations and recommendations from the Ecuadorian Andes. Mountain Research and Development 21: 77-83.

Ibero, C., C, Álvarez, J.C. Blanco, J. Criada, A. Sánchez \& C. Viada. 1996. Ríos de vida. Estado de conservación de las riberas fluviales en España. Sociedad Española de Ornitología, SEO/Birdlife. 45 pp.

Jáimez-Cuéllar, P., S. Vivas, N. Bonada, S. Robles, A. Mellado, M. Álvarez, J. Avilés, J. Casas, M. Ortega, I. Pardo, N. Prat, M. Rieradevall, C.E. Sáinz-Cantero, A. SánchezOrtega, M.L. Suárez, M. Toro, M.R. Vidal-Abarca, C. Zamora-Muñoz \& J. Alba-Tercedor. 2002. Protocolo GUADALMED (PRECE). Limnetica 21: 187-204.

Kutscher, A., C. Brand \& M.L. Miserendino. 2009. Evaluación de la calidad de los bosques de ribera en ríos del NO del Chubut sometidos a distintos usos de la tierra. Ecología Austral 19: 19-34.

LEDGER, R. 1968. Urban erosion in the USSR. Soviet Studies 19: 426-429.

Luebert, F. \& P. Pliscoff. 2006. Sinopsis bioclimática y vegetacional de Chile. Editorial Universitaria, Santiago de Chile. 316 pp.

Martínez, C. \& P. Lozano. 2004. Aplicación del índice de calidad del bosque de ribera, QBR al río Jucar en la provincia de Albacete. En: Instituto de estudios albacetenses "Don Juan Manuel" (eds.), Jornadas sobre el medio natural albacetense, pp. 313-321. II Jornadas Sobre el Medio Natural Albacetense, España.

Mitsch, W.J., R.H. Mitsch \& R.E. Turner. 1994. Wetlands of the old and new worlds: ecology and management. In: W.J. Mitsch (ed.) Global Wetlands old world and new, Elsevier, Amsterdam, the Netherlands. 53 pp.

MoreIRA, D.G. 2006. Metodologia de reabilitação fluvial integrada: O caso do rio estorãos na paisagem protegida das lagoas de Bertiandos E S. Pedro D’ Arcos. Dissertação para obtenção do grau de mestre em tecnología ambiental. Universidade de Tras-os-Montes e alto Douro, Portugal. 177 pp.

Munné, A., C. Solà \& N. Prat. 1998 a. QBR: Un índice rápido para la evaluación de la calidad de los ecosistemas de ribera. Tecnología del Agua 175: 20-37.

Munné, A., C. Solà, M. Rieradevall \& N. Prat. 1998 b. Índex QBR: Mètode per a l'avaluació de la qualitat dels ecosistemas de ribera. Estudis de la qualitat ecològica dels rius (4). Diputació de Barcelona, área de medi ambient. Barcelona, España. 28 pp.

Munné, A., N. Prat, C. Solà, N. Bonada \& M.R. Rieradevall. 2003. A simple field method for assessing the ecological quality of riparian habitat in rivers and streams: QBR index. Aquatic Conservation: Marine and Freshwater Ecosystems 13: 147-163.

MuotKa, T. \& P. LaAsonen. 2002. Ecosystem recovery in restored headwater stream: the role of enhanced leaf retention. Journal of Applied Ecology 39: 145-156.

Nahuelhual, L. \& D. NúÑEZ. 2011. Servicios ecosistémicos: contribución y desafíos para la conservación de la biodiversidad. En: J. Simonetti \& R. Dirzo (eds.), Conservación Biológica: Perspectivas desde América Latina, pp. 175-193. Editorial Universitaria, Santiago de Chile.

NorRIS, E.M. 2001. Riparian Restoration. Wetlands Program Technical Report. $\mathrm{N}^{\circ}$ 01-6.

Noss, R. 1990. Indicators for monitoring biodiversity: a hierarchical approach. Conservation Biology 4: 355-364.

Palma, A., R. Figueroa \& V.H. Ruiz. 2009. Evaluación de ribera y hábitat fluvial a través de los índices QBR e IHF. Gayana 73(1): 57-63.

Peña-Cortés, F., P. Gutiérrez, G. Rebolledo, M. Escalona, E. Hauenstein, C. Bertrán, R. Schlatter, \& J. Tapia. 2006. Determinación del nivel de antropización de humedales como criterio para la planificación ecológica de la cuenca del lago Budi, IX región de La Araucanía, Chile. Revista de Geografía Norte Grande (Chile) 36: 75-91.

Peredo, M., F. Martínez, E. Parada, D. Quevedo \& P. Olea. 2012. Informe final. Adaptación regional de un índice de estado para zonas riparianas y su aplicación en la cuenca del Maipo. Plataforma de investigación en ecohidrología y ecohidráulica. Santiago, Chile. 159 pp.

Pimentel, D. \& N. Kounang. 1998. Ecology of soil erosion in ecosystems. Ecosystems 1: 416-426.

Prat, N., M. Rieradevall, A. Munné, C. Solá \& N. Bonada. 1999. La qualitat ecológica del Llobregat, el Besós i el Foinx. Informe 1997. Estudis de la qualitat ecológica dels rius (6). Diputació de Barcelona. Àrea de medi ambient. Barcelona, España. 32 pp.

Quiroz, C.L., A. Pauchard, A. Marticorena \& L.A. Cavieres. 2009. Manual de plantas invasoras del centro-sur de Chile. Laboratorio de Invasiones Biológicas. Universidad de Concepción. 47 pp.

Rodríguez-Téllez, E., P.A. Domínguez-Calleros, M. PompaGarcía, J.A. Quiroz-Arratia \& M.E. Perez-López. 2012. Calidad del bosque de ribera del río El Tunal, Durango, México; mediante la aplicación del índice QBR. Gayana Botánica 69(1): 147-151.

Rzedowski, J. 1994. Vegetación de México. Ed. Limusa S.A., México. 432 pp.

Schade, J.D., E. Martí, J.R. Welter, S.G. Fisher \& N.B. Grimm. 2002. Sources of nitrogen to the riparian zone of a desert stream: implications for riparian vegetation and nitrogen retention. Ecosystems 5: 68-79.

Schade, J.D., S.G. Fisher, N.B. Grimm \& J.A. Seddon. 2001. The influence of a riparian shrub on nitrogen cycling in a Sonoran desert stream. Ecology 82(12): 3363-3376.

SHOwers, K.B. 1996. Soil erosion in the Kingdom of Lesotho and development of historical environmental impact assessment. Ecological Applications 6: 653-664.

SuÁrez, M.L. \& M.R. Vidal-AbarCA. 2000. Aplicación del índice de calidad del bosque de ribera, QBR a los cauces fluviales de la cuenca del río Segura. Tecnología del Agua 201: 3345.

Suárez, M.L., M.R. Vidal-Abarca, M.M. SÁnchez-Montoya, J. 
QBR en dos cuencas costeras del sur de Chile: CARRASco, S. ET AL.

Alba-Tercedor, M. Álvarez, J. Avilés, N. Bonada, J. Casas, P. Jáimez-Cuellar, A. Munné, I. Pardo, N. Prat, M. Rieradevall, M.J. Salinas, M. Toro \& S. Vivas. 2002. Las riberas de los ríos mediterráneos y su calidad: el uso del índice QBR. Limnetica 21: 135-148.

ToCKNER, K. \& J.V. WARD. 1999. Biodiversity along riparian corridors. Large rivers 11: 293-310.
TüZÜN, I. \& I. Albaryrak. 2005. The effect of disturbances to habitat quality on Otter (Lutra lutra) activity in the river Kizilirmak (Turkey): a case study. Turkish Journal of Zoology 29: 327-335.

WARD, J.V. 1998. Riverine landscapes: biodiversity patterns, disturbance regimes, and aquatic conservation. Conservation Biology 83: 269-278.

Recibido: 03.12.13

Aceptado: 11.02.14 